\title{
Maximum Entropy Interval Aggregations
}

\author{
Ferdinando Cicalese \\ Università di Verona, Verona, Italy \\ Email: cclfdn22@univr.it
}

\author{
Ugo Vaccaro \\ Università di Salerno, Salerno, Italy \\ Email: uvaccaro@unisa.it
}

\begin{abstract}
Given a probability distribution $\mathbf{p}=\left(p_{1}, \ldots, p_{n}\right)$ and an integer $1 \leq m<n$, we say that $\mathbf{q}=\left(q_{1}, \ldots, q_{m}\right)$ is a contiguous m-aggregation of $\mathbf{p}$ if there exist indices $0=i_{0}<$ $i_{1}<\cdots<i_{m-1}<i_{m}=n$ such that for each $j=1, \ldots, m$ it holds that $q_{j}=\sum_{k=i_{j-1}+1}^{i_{j}} p_{k}$. In this paper, we consider the problem of efficiently finding the contiguous $m$-aggregation of maximum entropy. We design a dynamic programming algorithm that solves the problem exactly, and two more time-efficient greedy algorithms that provide slightly sub-optimal solutions. We also discuss a few scenarios where our problem matters.
\end{abstract}

\section{INTRODUCTION}

The problem of aggregating data in a compact and meaningful way, and such that the aggregated data retain the maximum possible information contained in the original data, arises in many scenarios [8]. In this paper we consider the following particular instance of the general problem. Let $\mathcal{X}=\left\{x_{1}, \ldots, x_{n}\right\}$ be a finite alphabet, and $X$ be any random variable (r.v.) taking values in $\mathcal{X}$ according to the probability distribution $\mathbf{p}=\left(p_{1}, p_{2}, \ldots, p_{n}\right)$, that is, such that $P\left\{X=x_{i}\right\}=p_{i}>0$, for $i=1,2, \ldots, n$. Consider a partition $\Pi=\left(\Pi_{1}, \ldots, \Pi_{m}\right), m<n$, of the alphabet $\mathcal{X}$, where each class $\Pi_{i}$ of the partition $\Pi$ consists of consecutive elements of $\mathcal{X}$. That is, there exist indices $1 \leq i_{1}<\cdots<$ $i_{m-1}<i_{m}=n$ such that $\Pi_{1}=\left\{x_{1}, \ldots, x_{i_{1}}\right\}, \Pi_{2}=$ $\left\{x_{i_{1}+1}, \ldots, x_{i_{2}}\right\}, \ldots, \Pi_{m}=\left\{x_{i_{m-1}+1}, \ldots, x_{i_{m}}\right\}$. Any given such a partition $\Pi=\left(\Pi_{1}, \ldots, \Pi_{m}\right)$ naturally gives a r.v. $Y=f_{\Pi}(X)$, where for each $x \in \mathcal{X}$ it holds that $f_{\Pi}(x)=i$ if and only if $x \in \Pi_{i}$. Let $\mathbf{q}=\left(q_{1}, \ldots, q_{m}\right)$ be the probability distribution of r.v. $Y$. The values of the probabilities $q_{j}$ can obviously be computed as follows: for indices $0=i_{0}<i_{1}<$ $\cdots<i_{m-1}<i_{m}=n$ it holds that $q_{j}=\sum_{k=i_{j-1}+1}^{i_{j}} p_{k}$. The problem we consider in this paper is to determine the value

$$
\max _{\Pi} I\left(X ; f_{\Pi}(X)\right),
$$

where $I$ denotes the mutual information and the maximum is computed over all $m$-class partitions $\Pi=\left(\Pi_{1}, \ldots, \Pi_{m}\right)$ of set $\mathcal{X}$, in which each class $\Pi_{i}$ of the partition $\Pi$ consists of consecutive elements of $\mathcal{X}$. Since the function $f_{\Pi}$ is deterministic, the problem (1) can be equivalently stated as

$$
\max _{\Pi} H\left(f_{\Pi}(X)\right)
$$

where $H$ denotes Shannon entropy and the maximization takes place over the same domain as in (1). The formulation (1) is common in the area of clustering (e.g., [6], [10]) to emphasize that the objective is to reduce the "dimension" of the data (i.e., the cardinality of $|\mathcal{X}|$ ) under the constraint that the "reduced" data gives the maximum possible information towards the original, not aggregated data. We remark that, in general, there is no loss of generality in considering the problem (1) for deterministic functions only (e.g., see [9], [13]).

The contributions of this paper consist in efficient algorithms to solve the optimization problems (1) and (2). More precisely, we design a dynamic programming algorithm that runs in time $O\left(n^{2} m\right)$ to find a partition $\Pi$ that achieves the maximum in (2). Since the time complexity $O\left(n^{2} m\right)$ can be too large in some applications, we also provide much more efficient greedy algorithms that return a solution provably very close to the optimal one. We remark that the optimization problem (2) is strongly NP-hard in case the function $f$ is an arbitrary function such that $|f(\mathcal{X})|=m$, i.e., the partition into $m$ classes of $\mathcal{X}$ induced by $f$ is not constrained to contain only classes made by contiguous elements of $\mathcal{X}$ (see [3]).

The rest of the paper is organized as follows. In Section III we discuss the relevance of our results in the context of related works. In Section III we present our $O\left(n^{2} m\right)$ dynamic programming algorithm to solve problems (1) and (2). In the final Section IV we present two sub-optimal, but more time efficient, greedy algorithms for the same problems.

\section{RELATED WORK}

The problem of aggregating data (or source symbols, if we think of information sources) in an informative way has been widely studied in many different scenarios. One of the motivations is that data aggregation is often an useful, preliminary step to reduce the complexity of successive data manipulation. In this section we limit ourselves to point out the work that is strictly related to ours.

In the paper [12] the authors considered the following problem. Given a discrete memoryless source, emitting symbols from the alphabet $\mathcal{X}=\left\{x_{1}, \ldots, x_{n}\right\}$ according to the probability distribution $\mathbf{p}=\left(p_{1}, p_{2}, \ldots, p_{n}\right)$, the question is to find a partition $\Pi=\left(\Pi_{1}, \ldots, \Pi_{m}\right), m<n$, of the source alphabet $\mathcal{X}$ where, as before, each $\Pi_{i}$ consists of consecutive elements of $\mathcal{X}$, and such that the sum

$$
\frac{1}{m} \sum_{i=1}^{m} \sum_{j=1}^{m}\left|q_{i}-q_{j}\right|,
$$

is minimized. Each $q_{j}$ in (3) is the sum of the probabilities $p_{k}$ 's corresponding to the elements $x_{k} \in \mathcal{X}$ that belong to $\Pi_{j}$, that is our $q_{j}=\sum_{k=i_{j-1}+1}^{i_{j}} p_{k}$. The motivation of the authors of [12] to study above problem is that the minimization of 
expression (3) constitutes the basic step in the well known Fano algorithm [7] for $m$-ary variable length encoding finitealphabet memoryless source. In fact, solving (3) allows one to find a partition of $\mathcal{X}$ such that the cumulative probabilities of each class partition are as similar as possible. Obviously, the basic step has to be iterated in each class $\Pi_{i}$, till the partition is made by singletons. Now, it is not hard to see that

$$
\frac{1}{m} \sum_{i=1}^{m} \sum_{j=1}^{m}\left|q_{i}-q_{j}\right|=2+\frac{2}{m}-\frac{4}{m} \sum_{i=1}^{m} i q_{[i]},
$$

where $\left(q_{[1]}, \ldots, q_{[m]}\right)$ is the vector that contains the same elements as $\mathbf{q}=\left(q_{1}, \ldots, q_{m}\right)$, but now ordered in nonincreasing fashion. From equality (4) one can see that the problem of minimizing expression (3), over all partitions as stated above, is equivalent to maximizing the quantity $\sum_{i=1}^{m} i q_{[i]}$ over the same domain. The quantity $\sum_{i=1}^{m} i q_{[i]}$ is the well known guessing entropy by J. Massey [16]. Therefore, while in our problem (2) we seek a partition of $\mathcal{X}$ such that the cumulative probabilities of each class partition are as similar as possible, and the measure we use to appraise this quality is the Shannon entropy, the authors of [12] address the same problem using the guessing entropy, instead (this observation is not present in [12]). We should add that the criterion (3) used in [12] allows the authors to prove that the Fano algorithm produces an $m$-ary variable length encoding of the given source such that the average length of the encoding is strictly smaller than $\frac{H(\mathbf{p})}{\log m}+1-p_{\min }$, for $m=2$ and $m=3$ (and they conjecture that this is true also for any $m \geq 4$ ), where $\mathbf{p}$ is the source probability distribution and $p_{\text {min }}$ is the probability of the least likely source symbol. On the other hand, it is not clear how to efficiently solve the optimization problem (3). In fact, it is not known whether it enjoys or not the optimal substructure property, a necessary condition so that the problem could be optimally solved with known techniques like dynamic programming, greedy, etc. [5]. As mentioned before, our problem (2) can be optimally solved via dynamic programming. Numerical simulation suggests that optimal solutions to our problem (2) can be used to construct Fano encodings with the same upper bound on the average length as the ones constructed in [12].

A similar question, in which the aggregation operations of the elements of $\mathcal{X}$ are again constrained by given rules, was considered in [4]. There, the authors consider the problem of constructing the summary tree of a given weighted tree, by means of contraction operations on trees. Two types of contractions are allowed: 1) subtrees may be contracted to single nodes that represent the corresponding subtrees, 2) subtrees whose roots are siblings may be contracted to single nodes. Nodes obtained by contracting subtrees have weight equal to the sum of the node weights in the original contracted subtrees. Given a bound on the number of nodes in the resulting summary tree, the problem is to compute the summary tree of maximum entropy, where the entropy of a tree is the Shannon entropy of the normalized node weights. In [18] the authors consider the problem of quantizing a finite alphabet $\mathcal{X}$ by collapsing properly chosen contiguous sequences of symbols of $\mathcal{X}$ (called convex codecells in [18]) to single elements. The objective is to minimize the expected distortion induced by the quantizer, for some classes of distortion measures. Our similar scenario would correspond to the minimization of $H(X)-H\left(f_{\Pi}(X)\right)$, not considered in [18].

Our results could find applications also in data compression for sources with large alphabet (e.g. [17]). One could use our techniques as a pre-processing phase to reduce the source alphabet from a large one to a smaller one, in order to obtain a new source that retains most of the entropy as the original one, just because of (2). An encoding of the so constructed "reduced source" can be easily transformed to an encoding of the original source by exploiting the fact that the partition of the original source alphabet has been performed with consecutive subsets of symbols. Finally, other problems similar to ours were considered in papers [11], [14]. It seems that our findings could be useful in "histogram compression", where the constraint that one can merge only adjacent class intervals is natural [19].

\section{AN OPTIMAL DYNAMIC PROGRAMMING ALGORITHM}

We find it convenient to formulate problems (1) and (2) in a slightly different language. We give the following definition.

Definition 1. Given a n-dimensional vector of strictly positive numbers $\mathbf{p}=\left(p_{1}, \ldots, p_{n}\right)$ and a positive integer $m<n$, we say that a vector $\mathbf{q}=\left(q_{1}, \ldots, q_{m}\right)$ is a contiguous $m$ aggregation of $\mathbf{p}$ if the following condition hold: there exist indices $0=i_{0}<i_{1}<\cdots<i_{m-1}<i_{m}=n$ such that for each $j=1, \ldots, m$ it holds that $q_{j}=\sum_{k=i_{j-1}+1}^{i_{j}} p_{k}$.

Thus, our problems can be so formulated:

Problem Definition. Given an $n$-dimensional probability distribution $\mathbf{p}=\left(p_{1}, \ldots, p_{n}\right)$ (where all components are assumed to be strictly positive) and an integer $1 \leq m<n$, find a contiguous $m$-aggregation of $\mathbf{p}$ of maximum entropy.

Our dynamic programming algorithm proceeds as follows. For $j=1, \ldots, n$, let $s_{j}=\sum_{k=1}^{j} p_{k}$. Notice that we can compute all these values in $O(n)$ time. For a sequence of numbers $\mathbf{w}=w_{1}, \ldots, w_{t}$ such that for each $i=1, \ldots, t, w_{i} \in(0,1]$ and $\sum_{i=1}^{t} w_{i} \leq 1$, we define the entropy-like sum of $\mathbf{w}$ as $\tilde{H}(\mathbf{w})=-\sum_{j=1}^{t} w_{t} \log w_{t}$. Clearly when $\mathbf{w}$ is a probability distribution we have that the entropy-like sum of $\mathbf{w}$ coincides with the Shannon entropy of $\mathbf{w}$. For each $i=1, \ldots, m$ and $j=1, \ldots, n$ let $h q[i, j]$ be the maximum entropy-like sum of a contiguous $i$-aggregation of the sequence $p_{1}, \ldots, p_{j}$. Therefore, $h q[m, n]$ is the sought maximum entropy of a contiguous $m$-aggregation of $\mathbf{p}$. Let $\hat{\mathbf{q}}=\left(q_{1}, \ldots, q_{i}\right)$ be a contiguous $i$-aggregation of $\left(p_{1}, \ldots, p_{j}\right)$ of maximum entropylike sum. Let $r$ be the index such that $q_{i}=\sum_{k=r}^{j} p_{k}$. We have $q_{i}=s_{j}-s_{r-1}$ and

$$
\tilde{H}(\hat{\mathbf{q}})=-\left(s_{j}-s_{r-1}\right) \log \left(s_{j}-s_{r-1}\right)+\tilde{H}\left(\mathbf{q}^{\prime}\right),
$$

where $\mathbf{q}^{\prime}=\left(q_{1}, \ldots, q_{i-1}\right)$. Now we observe that $\mathbf{q}^{\prime}$ is a contiguous $(i-1)$-aggregation of $\left(p_{1}, \ldots, p_{r-1}\right)$. Moreover, 
since $\tilde{H}(\hat{\mathbf{q}})$ is maximum-among the entropy-like sum of any contiguous $i$-aggregation of $\left(p_{1}, \ldots, p_{i}\right)$ - it must also hold that $\tilde{H}\left(\mathbf{q}^{\prime}\right)$ is maximum among any contiguous $(i-1)$ aggregation of $\left(p_{1}, \ldots, p_{r-1}\right)$. Based on this observation we can compute the $h q[\cdot, \cdot]$ values recursively as follows:

$h q[i, j]= \begin{cases}\max _{k=i, \ldots, j}\{h q[i-1, k-1] & \\ \left.-\left(s_{j}-s_{k-1}\right) \log \left(s_{j}-s_{k-1}\right)\right\} & i>1, j \geq i \\ -s_{j} \log s_{j} & i=1 .\end{cases}$

There are $n \times m$ values to be computed and each one of them can be computed in $O(n)$ (due to the max in the first case). Therefore the computation of $h[m, n]$ requires $O\left(n^{2} m\right)$ time. By a standard procedure, once one has the whole table $h q[\cdot, \cdot]$, one can reconstruct the contiguous $m$-aggregation of $\mathbf{p}$ achieving entropy $h q[m, n]$ by backtracking on the table.

\section{Sub-OPTIMAL GREEDY ALGORITHMS}

We start by recalling a few notions of majorization theory [15] that are relevant to our context.

Definition 2. Given two probability distributions $\mathbf{a}=$ $\left(a_{1}, \ldots, a_{n}\right)$ and $\mathbf{b}=\left(b_{1}, \ldots, b_{n}\right)$ with $a_{1} \geq \ldots \geq a_{n} \geq 0$ and $b_{1} \geq \ldots \geq b_{n} \geq 0, \sum_{i=1}^{n} a_{i}=\sum_{i=1}^{n} b_{i}=1$, we say that $\mathbf{a}$ is majorized by $\mathbf{b}$, and write $\mathbf{a} \preceq \mathbf{b}$, if and only if $\sum_{k=1}^{i} a_{k} \leq \sum_{k=1}^{i} b_{k}, \quad$ for all $i=1, \ldots, n$.

We use the majorization relationship between vectors of unequal lengths, by properly padding the shorter one with the appropriate number of 0 's at the end. Majorization induces a lattice structure on $\mathcal{P}_{n}=\left\{\left(p_{1}, \ldots, p_{n}\right)\right.$ : $\sum_{i=1}^{n} p_{i}=$ $\left.1, p_{1} \geq \ldots \geq p_{n} \geq 0\right\}$, see [1]. Shannon entropy function enjoys the important Schur-concavity property [15]: For any $\mathbf{x}, \mathbf{y} \in \mathcal{P}_{n}, \mathbf{x} \preceq \mathbf{y}$ implies that $H(\mathbf{x}) \geq H(\mathbf{y})$. We also need the concept of aggregation and a result from [2]. Given $\mathbf{p}=\left(p_{1}, \ldots, p_{n}\right) \in \mathcal{P}_{n}$ and an integer $1 \leq m<n$, we say that $\mathbf{q}=\left(q_{1}, \ldots, q_{m}\right) \in \mathcal{P}_{m}$ is an aggregation of $\mathbf{p}$ if there is a partition of $\{1, \ldots, n\}$ into disjoint sets $I_{1}, \ldots, I_{m}$ such that $q_{j}=\sum_{i \in I_{j}} p_{i}$, for $j=1, \ldots m$.

Lemma 1. [2] Let $\mathbf{q} \in \mathcal{P}_{m}$ be any aggregation of $\mathbf{p} \in \mathcal{P}_{n}$. Then it holds that $\mathbf{p} \preceq \mathbf{q}$.

We now present our first greedy approximation algorithm for the problem of finding the maximum entropy contiguous $m$-aggregation of a given probability distribution $\mathbf{p}=$ $\left(p_{1}, \ldots, p_{n}\right)$. The pseudocode of the algorithm is given below.

The algorithm has two phases. In the first phase, lines from 2 to 9 the algorithm iteratively builds a new component of $\mathbf{q}$ as follows: Assume that the first $i$ components of $\mathbf{q}$ have been produced by aggregating the first $j$ components of p. If $p_{j+1}>2 / m$ then $q_{i+1}$ is the aggregation of the singleton interval containing only $p_{j+1}$. Otherwise, $q_{i+1}$ is set to be the aggregation of the largest number of components $p_{j+1}, p_{j+2}, \ldots$ such that their sum is not larger than $2 / \mathrm{m}$.

For each $k=1, \ldots, i$, the values $\operatorname{start}[k]$ and $\operatorname{end}[k]$ are meant to contain the first and the last component of $\mathbf{p}$

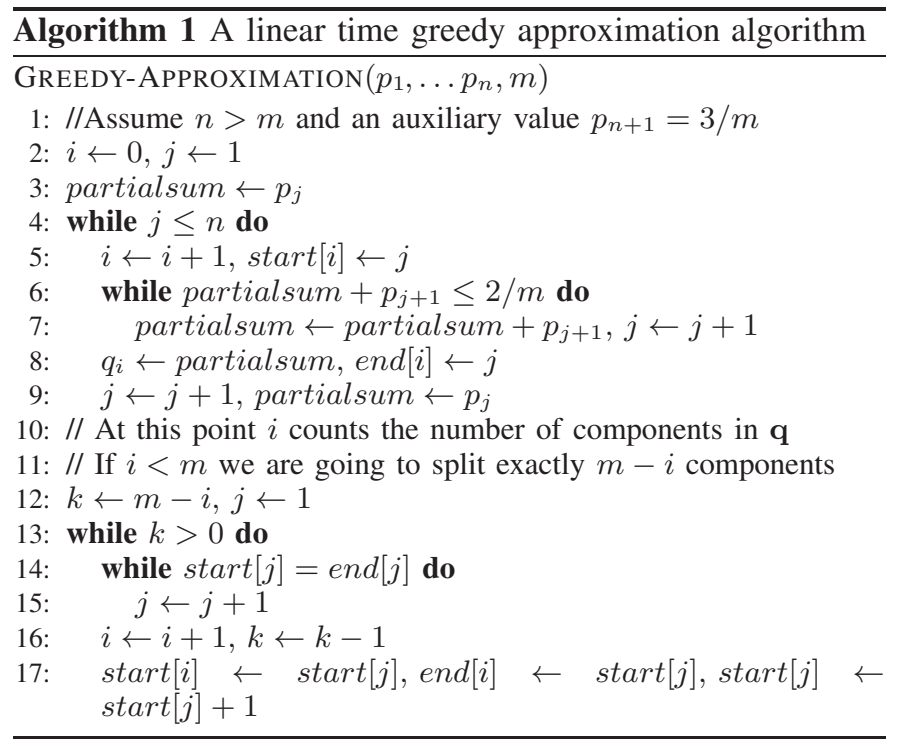

which are aggregated into $q_{k}$. By construction, we have that $\operatorname{start}[k] \neq \operatorname{end}[k]$ indicates that $q_{k} \leq 2 / m$. The first crucial observation is that, at the end of the first phase, the number $i$ of components in the distribution $\mathbf{q}$ under construction is smaller than $m$. To see this, it is enough to observe that by construction $q_{j}+q_{j+1}>2 / m$, for any $j=1,2, \ldots,\lfloor i / 2\rfloor$. Therefore, arguing by contradiction, if we had $i \geq m+1$ we would reach the following counterfactual inequality

$1=\sum_{j=1}^{i} q_{j} \geq \sum_{j=1}^{\lfloor i / 2\rfloor}\left(q_{2 j-1}+q_{2 j}\right)>\sum_{j=1}^{\lfloor i / 2\rfloor} \frac{2}{m}=\left\lfloor\frac{i}{2}\right\rfloor \frac{2}{m} \geq \frac{2 i-2}{2 m} \geq 1$.

In the second phase, lines 12,17, the algorithm splits the first $m-i$ components of $\mathbf{q}$ which are obtained by aggregating at least two components of $\mathbf{p}$. Notice that, as observed above, such components of $\mathbf{q}$ are not larger than $2 / m$. Hence, also the resulting components in which they are split have size at most $2 / m$. It is important to notice that there must exist at least $m-i$ such "composite' 1 components, because of the assumption $n>m$, and the fact that each component of $\mathbf{p}$ is non zero. As a result of the above considerations, the aggregation $\mathbf{q}$ returned by the GREEDY-APPROXIMATION algorithm can be represented, after reordering its components in non-increasing order, as $\mathbf{q}=\left(q_{1}, \ldots, q_{k^{*}}, q_{k^{*}+1}, \ldots q_{m}\right)$, where $q_{1}, \ldots, q_{k^{*}}$ are all larger than $2 / m$ and coincide with the $k^{*}$ largest components of $\mathbf{p}$ and the remaining components of $\mathbf{q}$, namely $q_{k^{*}+1}, \ldots, q_{m}$, are all not larger than $2 / m$. Let us now define the quantities $A=1-\sum_{j=1}^{k^{*}} q_{j}, \quad$ and $B=\sum_{j=1}^{k^{*}} q_{j} \log \frac{1}{q_{j}}$. It holds that

$$
\begin{aligned}
H(\mathbf{q}) & =\sum_{j=1}^{k^{*}} q_{j} \log \frac{1}{q_{j}}+\sum_{j=k^{*}+1}^{m} q_{j} \log \frac{1}{q_{j}} \\
& =B+\sum_{j=k^{*}+1}^{m} q_{j} \log \frac{1}{q_{j}}
\end{aligned}
$$

${ }^{1}$ We are calling a component $q_{j}$ composite if it is obtained as the sum of at least two components of $\mathbf{p}$. 


$$
\begin{aligned}
& \geq B+\sum_{j=k^{*}+1}^{m} q_{j} \log \frac{m}{2} \\
& =B+A \log (m)-A
\end{aligned}
$$

where (6) follows by definition of $B$; (7) follows by the fact that $q_{j} \leq \frac{2}{m}$ for any $j>k^{*} ; 8$ follows by definition of $A$ and the basic properties of the logarithm.

Lemma 2. Let $\tilde{\mathbf{q}}$ be the probability distribution defined as $\tilde{\mathbf{q}}=\left(q_{1}, \ldots, q_{k^{*}}, \frac{A}{m-k^{*}}, \ldots, \frac{A}{m-k^{*}}\right)$. Then, it holds that:

$$
H(\mathbf{q}) \geq H(\tilde{\mathbf{q}})-\frac{2}{e \ln (2)} .
$$

Proof. We have

$$
\begin{aligned}
H(\tilde{\mathbf{q}}) & =\sum_{j=1}^{k^{*}} q_{j} \log \frac{1}{q_{j}}+\sum_{j=k^{*}+1}^{m} \frac{A}{m-k^{*}} \log \frac{m-k^{*}}{A} \\
& =B+A \log \left(m-k^{*}\right)-A \log A .
\end{aligned}
$$

Therefore, by using the above lower bound (5)-(8) on the entropy of $\mathbf{q}$ it follows that

$$
\begin{aligned}
H(\tilde{\mathbf{q}})-H(\mathbf{q}) & \leq A \log \frac{m-k^{*}}{m}-A \log (A)+A \\
& \leq-A \log (A)+A \leq \frac{2}{e \ln (2)}
\end{aligned}
$$

where the second inequality follows since $A \log \frac{m-k^{*}}{m} \leq 0$ for any $k^{*} \geq 0$ and the last inequality follows by the fact that $A \in[0,1]$ and the maximum of the function $-x \log x+x$ in the interval $[0,1]$ is $\frac{2}{e \ln (2)}$.

Let $\mathbf{q}^{*}=\left(q_{1}^{*}, \ldots q_{m}^{*}\right)$ be a contiguous $m$-aggregation of $\mathbf{p}$ of maximum entropy. We can use $\tilde{\mathbf{q}}$ to compare the entropy of our greedily constructed contiguous $m$-aggregation $\mathbf{q}$ to the entropy of $\mathbf{q}^{*}$. We prepare the following

Lemma 3. It holds that $\tilde{\mathbf{q}} \preceq \mathbf{q}^{*}$, therefore $H(\tilde{\mathbf{q}}) \geq H\left(\mathbf{q}^{*}\right)$.

Proof. Assume, w.l.o.g., that the components of $\mathbf{q}^{*}$ are sorted in non-increasing order. Let $\tilde{\mathbf{p}}=\left(\tilde{p}_{1}, \ldots, \tilde{p}_{n}\right)$ be the probability distribution obtained by reordering the components of $\mathbf{p}$ in non-increasing order. It is not hard to see that, by construction, we have $\tilde{p}_{j}=q_{j}$ for each $j=1, \ldots, k^{*}$. Since $\mathbf{q}^{*}$ is an aggregation of $\tilde{\mathbf{p}}$, by Lemma 1, we have that $\tilde{\mathbf{p}} \preceq \mathbf{q}^{*}$, which immediately implies

$$
\sum_{s=1}^{j} q_{s}=\sum_{s=1}^{j} \tilde{p}_{s} \leq \sum_{s=1}^{j} q_{j}^{*} \quad \text { for each } j=1, \ldots, k^{*} .
$$

Moreover, by the last inequality with $j=k^{*}$ it follows that $\sum_{s=k^{*}+1}^{m} q_{s}^{*} \leq 1-\sum_{s=1}^{k^{*}} q_{s}=A$. This, together with the assumption that $q_{1}^{*} \geq \cdots q_{k^{*}}^{*} \geq q_{k^{*}+1}^{*} \geq \cdots q_{m}^{*}$ implies that

$$
\sum_{s=t+1}^{m} q_{s}^{*} \leq \frac{m-t}{m-k^{*}} A \quad \text { for any } t \geq k^{*} .
$$

Then, for each $j=k^{*}, \ldots, m$ we have

$\sum_{s=1}^{j} q_{j}^{*}=1-\sum_{s=j+1}^{m} q_{s}^{*} \geq 1 \frac{m-j}{m-k^{*}} A=1-\sum_{s=j+1} m \tilde{q}_{s}=\sum_{s=1}^{j} \tilde{q}_{s}$ that together with (9) implies $\tilde{\mathbf{q}} \preceq \mathbf{q}^{*}$.

This concludes the proof of the first statement of the Lemma. The second statement immediately follows by the Schur concavity of the entropy function.

We are now ready to summarize our findings.

Theorem 1. Let $\mathbf{q}$ be the contiguous m-aggregation of $\mathbf{p}$ returned by GREEDY-APPROXIMATION. Let $\mathbf{q}^{*}$ be a contiguous m-aggregation of $\mathbf{p}$ of maximum entropy. Then, it holds that

$$
H(\mathbf{q}) \geq H\left(\mathbf{q}^{*}\right)-\frac{2}{e \ln (2)}=H\left(\mathbf{q}^{*}\right)-1.0614756 \ldots
$$

Proof. Directly from Lemmas 3 and 2.

\section{A. A slightly improved greedy approach}

We can improve the approximation guarantee of Algorithm 1 by a refined greedy approach of complexity $O(n+m \log m)$. The new idea is to build the components of $\mathbf{q}$ in such a way that they are either not larger than $3 / 2 m$ or they coincide with some large component of $\mathbf{p}$. More precisely, when building a new component of $\mathbf{q}$, say $q_{i}$, the algorithm puts together consecutive components of $\mathbf{p}$ as long as their sum, denoted partialsum, is not larger than $1 / \mathrm{m}$. If, when trying to add the next component, say $p_{j}$, the total sum becomes larger than $1 / m$ the following three cases are considered:

Case 1. partialsum $+p_{j} \in\left[\frac{1}{m}, \frac{3}{2 m}\right]$.

In this case $q_{i}$ is set to include also $p_{j}$ hence becoming a component of $\mathbf{q}$ of size not larger than $3 / 2 \mathrm{~m}$.

Case 2. partialsum $+p_{j}>\frac{2}{m}$.

In this case we produce up to two components of $\mathbf{q}$. Precisely, if partialsum $=0$ that is $p_{j}>2 / m$ we set $q_{i}=p_{j}$ and only one new component is created. Otherwise, $q_{i}$ is set to partialsum (i.e., it is the sum of the interval up to $p_{j-1}$, and it is not larger than $1 / m$ and $q_{i+1}$ is set to be equal to $p_{j}$. Notice that in this case $q_{i+1}$ might be larger than $3 / 2 m$ but it is a non-composite component.

Case 3. partialsum $+p_{j} \in\left(\frac{3}{2 m}, \frac{2}{m}\right)$.

In this case we produce one component of $\mathbf{q}$, namely $q_{i}$ is set to partialsum $+p_{j}$ and we mark it.

We first observe that the total number of components of $\mathbf{q}$ created by this procedure is not larger than $m$. More precisely, let $k_{1}, k_{2}, k_{3}$ be the number of components created by the application of Case 1, 2, and 3 respectively. Each component created by Case 1 has size $\geq 1 / m$. When we apply Case 2 we create either one component of size $>2 / m$ or two components of total sum $>2 / m$. Altogether the $k_{2}$ components created by Case 2 have total sum at least $k_{2} / \mathrm{m}$. Then, since each component created by applying Case 3 has size at least $3 / 2 m$ we have that $k_{3} \leq \frac{1-\left(k_{1}+k_{2}\right) / m}{3 / 2 m}=\frac{2\left(m-k_{1}-k_{2}\right)}{3}$, hence $k_{1}+k_{2}+k_{3} \leq \frac{2 m}{3}+\frac{1}{3}\left(k_{1}+k_{2}\right)$, from which we get 1) $m-k_{1}-k_{2} \geq \frac{3}{2} k_{3}$, and 2) $m-k_{1}-k_{2}-k_{3} \geq \frac{1}{2} k_{3}$. Inequalities 1) and 2) mean that if $k_{3}>0$ then the number of components created is smaller than $m$ by a quantity which equals at least half of $k_{3}$. In other words, we are allowed to split at least half of the $k_{3}$ components created by Case 3 and the resulting total number of components will still be not larger than $m$. In the 
second phase of the algorithm, the largest components created from Case 3 are split. As a result of the above considerations, the final distribution $\mathbf{q}$ returned by the algorithm has: (i) components $>2 / m$ which are singletons, i.e., coincide with components of $\mathbf{p}$; the remaining components can be divided into two sets, the components of size $>3 / 2 m$ and the ones of size $\leq 3 / 2 m$ with the second set having larger total probability mass. In formulas, we can represent the probability vector $\mathbf{q}$, after reordering its components in non-increasing order, as $\mathbf{q}=\left(q_{1}, \ldots, q_{k^{*}}, q_{k^{*}+1}, \ldots q_{j^{*}}, q_{j^{*}+1}, \ldots, q_{m}\right)$, where: (i) $q_{1}, \ldots, q_{k^{*}}$ are all larger than $2 / m$ and coincide with the $k^{*}$ largest components of $\mathbf{p}$; (ii) $q_{k^{*}+1}, \ldots, q_{j^{*}}$ are all in the interval $(3 / 2 m, 2 / m)$; (iii) $q_{j^{*}+1}, \ldots, q_{m}$, are all not larger than $3 / 2 m$. Let us define the quantities

$$
A_{1}=\sum_{s=k^{*} 1}^{j^{*}} q_{s}, \quad A_{2}=\sum_{s=j^{*} 1}^{m} q_{s}, \quad B=\sum_{j=1}^{k^{*}} q_{j} \log \frac{1}{q_{j}} .
$$

Let $A=A_{1}+A_{2}$. Since the algorithm splits the largest components of size $3 / 2 m$ it follows that $A_{2} \geq A / 2$. Then, by proceeding like in the previous section we have

$$
\begin{aligned}
H(\mathbf{q}) & =\sum_{s=1}^{k^{*}} q_{s} \log \frac{1}{q_{s}}+\sum_{s=k^{*}+1}^{j^{*}} q_{s} \log \frac{1}{q_{s}}+\sum_{s=j^{*}+1}^{m} q_{s} \log \frac{1}{q_{s}} \\
& \geq B+\sum_{s=k^{*}+1}^{j^{*}} q_{s} \log \frac{m}{2}+\sum_{s=j^{*}+1}^{m} q_{s} \log \frac{2 m}{3} \\
& =B+\left(A_{1}+A_{2}\right) \log (m)-A_{1}-A_{2} \log \frac{3}{2} \\
& \geq B+A \log (m)-\frac{A}{2} \log (3)
\end{aligned}
$$

where the last inequality holds since $A_{2} \geq A / 2$. Proceeding like in Lemma 2 above, we have the following result.

Lemma 4. Let $\tilde{\mathbf{q}}$ be the probability distribution defined as $\tilde{\mathbf{q}}=\left(q_{1}, \ldots, q_{k^{*}}, \frac{A}{m-k^{*}}, \ldots, \frac{A}{m-k^{*}}\right)$. It holds that:

$$
H(\mathbf{q}) \geq H(\tilde{\mathbf{q}})-\frac{\sqrt{3}}{e \ln (2)} .
$$

This result, together with Lemma 3 implies

Theorem 2. Let $\mathbf{q}$ be the contiguous m-aggregation of $\mathbf{p}$ returned by the algorithm GREEDY-2. Let $\mathbf{q}^{*}$ be a contiguous $m$-aggregation of $\mathbf{p}$ of maximum entropy. Then, it holds that

$$
H(\mathbf{q}) \geq H\left(\mathbf{q}^{*}\right)-\frac{\sqrt{3}}{e \ln (2)}=H\left(\mathbf{q}^{*}\right)-0.91926 \ldots .
$$

\section{REFERENCES}

[1] F. Cicalese and U. Vaccaro, "Supermodularity and subadditivity properties of the entropy on the majorization lattice", IEEE Transactions on Information Theory, Vol. 48, 933-938, 2002.

[2] F. Cicalese, L. Gargano, and U. Vaccaro, " $H(X)$ vs. $H(f(X))$ ", in: Proc. ISIT 2017, pp. 51-55.

[3] F. Cicalese L. Gargano, and U. Vaccaro, "Bounds on the Entropy of a Function of a Random Variable and their Applications", IEEE Transactions on Information Theory, Vol. 64, 2220-2230, 2018.

[4] R. Cole and H. Karloff,"Fast algorithms for constructing maximum entropy summary trees", in: Proc. of ICALP 2014, pp. 332-343, 2014.

[5] T.H. Cormen, C.E. Leiserson, R.L. Rivest, C. Stein, Introduction to Algorithms, MIT Press, (2009).

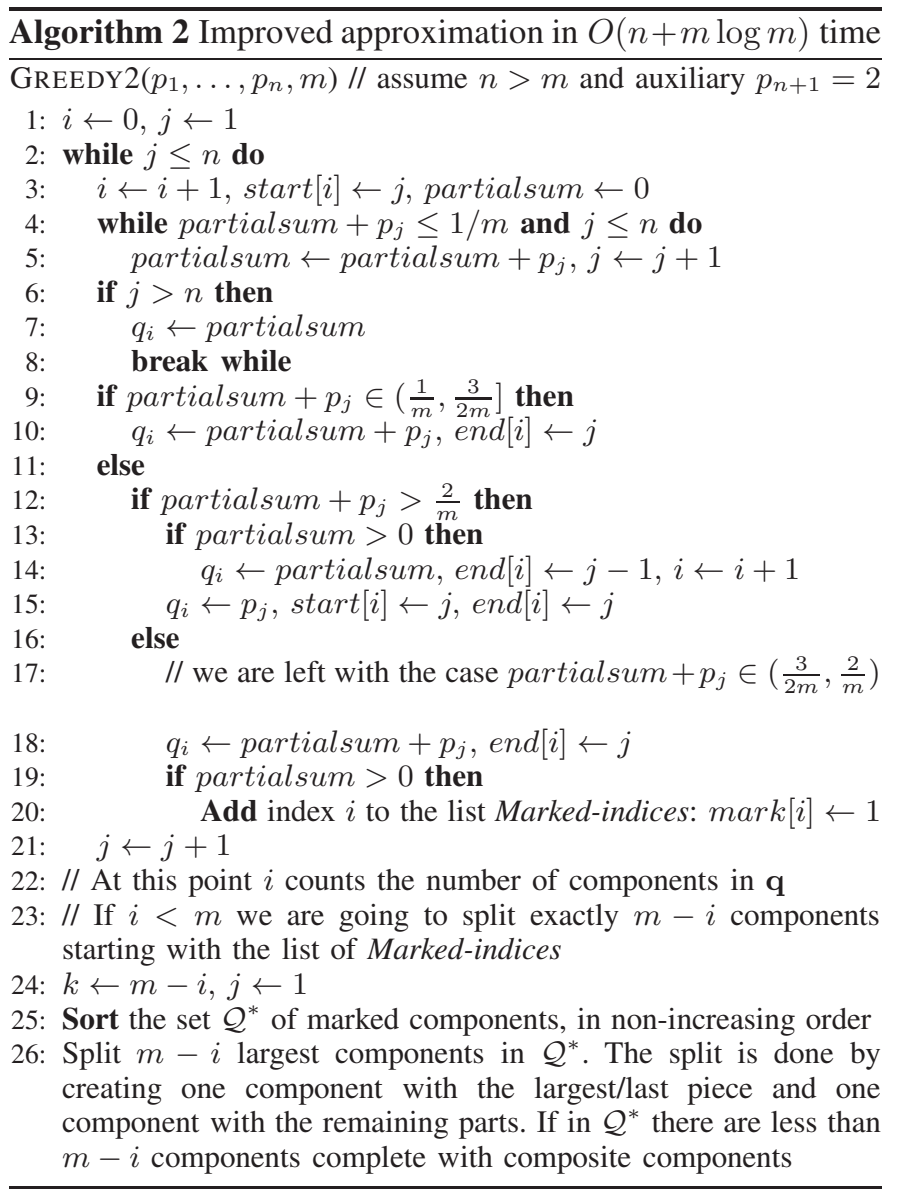

[6] L. Faivishevsky and J. Faivishevsky, "Nonparametric information theoretic clustering algorithm", in: Proceedings of the 27th International Conference on Machine Learning (ICML-10), pp. 351-358, 2010.

[7] R. M. Fano, "The transmission of information", Research Laboratory of Electronics, Mass. Inst. of Techn. (MIT), Tech. Report No. 65, 1949.

[8] G. Gan, C. Ma, and J. Wu, Data Clustering: Theory, Algorithms, and Applications, ASA-SIAM Series on Statistics and Applied Probability, SIAM, Philadelphia, ASA, Alexandria, VA, 2007.

[9] B.C. Geiger and R.A. Amjad, "Hard Clusters Maximize Mutual Information", arXiv:1608.04872 [CS.IT], 2016.

[10] M. Kearns, Y. Mansour, and A. Y. Ng, "An information-theoretic analysis of hard and soft assignment methods for clustering." In: Learning in graphical models. Springer Netherlands, pp. 495-520, 1998.

[11] T. Kampke and R. Kober, "Discrete signal quantizations", Pattern Recognition, vol. 32, pp. 619-634, (1999).

[12] S. Krajči, C.-F. Liu, L. Mikeš, and S.M. Moser, "Performance analysis of Fano coding", in: Proc. of ISIT 2015, pp. 1746-1750.

[13] B.M. Kurkoski, H. Yagi, "Quantization of Binary-Input Discrete Memoryless Channels", IEEE Trans. on Inf. Theory, 60, 4544-4552, 2014.

[14] R. Lamarche-Perrin, Y. Demazeau, J.-M. Vincent, "The best-partitions problem: How to build meaningful aggregations", 2013 IEEE/WIC/ACM Inter. Conf. on Web Intell. and Intell. Agent Techhology, 309-404, 2013.

[15] A.W. Marshall, I. Olkin, B. C. Arnold, Inequalities: Theory of Majorization and Its Applications, Springer, New York (2009).

[16] J.L. Massey, "Guessing and entropy", in: Proc. of 1994 IEEE International Symposium on Information Theory, Trondheim, Norway, p. 204.

[17] A. Moffat and A. Turpin, "Efficient construction of minimumredundancy codes for large alphabets", IEEE Transactions on Information Theory, vol. 44, 1650-1657, 1998.

[18] D. Muresan and M. Effros, "Quantization as Histogram Segmentation: Optimal Scalar Quantizer Design in Network Systems", IEEE Transactions on Information Theory, Vol. 54, 344-366 (2008).

[19] G.M. Perillo, E. Marone, "Determination of optimal number of class intervals using maximum entropy", Math. Geo., 18, 401-407, 1986. 